\title{
LIPIDOMICS: ADVANCED ANALYTICAL TECHNOLOGY TO IDENTIFY BIOMARKERS OF COLORECTAL CANCER
}

\author{
CLAUDIU RĂCHIERIU ${ }^{a, b}$, DAN T. ENIU b,c, \\ EMIL MOIŞ ${ }^{b, d}, F^{2} O R I N ~ G R A U R^{b, d}$, CARMEN SOCACIU ${ }^{*}$, \\ MIHAI ADRIAN SOCACIUf, NADIM ALHAJJAR ${ }^{\text {b,d }}$
}

\begin{abstract}
Lipidomics is an advanced analytical technology applying techniques like Gas- or Liquid Chromatography coupled with Mass Spectrometry or Magnetic Resonance Spectrometry to find the lipid biomarkers in tissues, blood or urine. This review selected significant results which show the impact of lipidomic investigations for discriminating the metabolic profiles and dysregulation of metabolic pathways in CRC patient's comparative to healthy controls. It focuses on the description of best methods and procedures to separate and identify hydrophobic molecules with molecular weights under 1000 Daltons, especially using high pressure liquid chromatography coupled with mass spectrometry. The main lipid classes involved in the onset and progression of CRC are presented, as well the alterations of lipid-related cellular pathways during tumorigenesis. Especially lipid profile changes (of cerotic acid, hydroxylated, polyunsaturated fatty acids, free fatty acids, oxylipins, glycerophospholipids, di- and triglycerides, sphingomyelins and ceramides) showed significant differences and can be considered reliable biomarkers to discriminate between early and advanced stages of CTC malignancy, prognosis or survival prediction.
\end{abstract}

Keywords: metabolomics, lipidomics, colorectal cancer, biomarkers.

a Surgery Department. County Hospital Alba, Bld. Revolutiei 1989 No.23, Alba Iulia, Romania

b Department of Oncological Surgery and Gynecological Oncology, "Iuliu Hațieganu" University of Medicine and Pharmacy, 34-36 Republicii Str, 400015 Cluj-Napoca, Romania

c Department of Surgery, The Oncology Institute "Prof. Dr. Ion Chiricuță", Cluj-Napoca

d Faculty of Medicine, Iuliu Hatieganu University of Medicine and Pharmacy, Cluj-Napoca, Romania; Surgery Department, Regional Institute of Gastroenterology and Hepatology "Octavian Fodor", 19-21 Croitorilor Street, Cluj-Napoca, Romania

e R\&D Center for Applied Biotechnology in Diagnosis and Molecular Therapy, BIODIATECHProplanta Cluj-Napoca, Romania

f Faculty of Medicine, Iuliu Hatieganu University of Medicine and Pharmacy, Cluj-Napoca, Romania; Radiology Department, Regional Institute of Gastroenterology and Hepatology "Octavian Fodor", 19-21 Croitorilor Street, Cluj-Napoca, Romania

*Corresponding author: csocaciu@proplanta.ro 


\section{Abbreviations}

Cer: Ceramide; CEA: carcinoembryonic antigen; CRC: Colorectal cancer; CTC: computed tomography colonography; FFA: Free fatty acid; GTA: Gastrointestinal tract acids; HETE: Hydroxyeicosatetraenoic acid; HODE: 13-hydroxyoctadecadienoic acid; LA: Linoleic acid; Lysophosphatidic acid; LOX- lipoxygenase; LPC: Lysophosphatidylcholine; MUFA: Monounsaturated fatty acid; PA: Phosphatidic acid PC: Phosphatidylcholine; PE: Phosphorylethanolamine; PG: Prostaglandin; PL: Phospholipid; PUFA: Polyunsaturated fatty acid; RBC: Red blood cell; SFA: Saturated fatty acid; SM: Sphingomyelin; SPL: Sphingolipid; TAG: Triacylglycerol;

\section{INTRODUCTION}

Colorectal Cancer (CRC) is an important public health issue, belongs to the three leading causes of cancer-related mortality in both men and women [1,2], particularly in Western Countries but also in developing countries, and is strongly related to stress and food habits. The American Cancer Society estimated 104610 cases in 2020 , and other 43,340 new cases in US, around $12 \%$ diagnosed in patients less than 50 years old [3]. The incidence is $30 \%$ higher in men compared to women with an increased incidence $(60 \%)$ for rectal cancer, compared to colon cancer. The early detection and endoscopic resection of adenomatous polyps (premalignant conditions) and screening colonoscopy improved significantly the survival rate (American Cancer Society, 2020). The decline of mortality during the last two decades are due to improvements in treatment (12\%), changes in CRC risk factors (35\%), and screening (53\%) as well as due to early diagnosis, with a CRC decline of $64 \%$ after 5 years and $58 \%$ after 10 years [4].

Recent data compared different ways to diagnose CRC, many including a preliminary colonoscopy and biopsy [5-7] and more and more, imaging technologies [8-10].

Blood is particularly useful for biomarker research, as it can be obtained more easily and less invasively than other biological materials. That is the reason why scientists are searching for other screening methods with a good predictive value and high sensitivity. Metabolomics is an advanced non-invasive technology which includes a systematic screening, fingerprinting of small metabolites (with molecular weight less than $5000 \mathrm{Da}$ ) which are related to the metabolic signature of a certain tissue or biofluid (blood, urine, saliva).

Metabolomics and metabonomics offer a qualitative description (fingerprint) and the quantitative measurement, respectively. If the screening refers specifically to lipid molecules involved in metabolism, this screening is named Lipidomics, which include two types of techniques: untargeted lipidomics, 
a comprehensive analysis of all the measurable lipid molecules in a sample including unknown chemicals, and targeted lipidomics, which measure defined groups of known lipids, that are characterized and chemically annotated [11, 12]. This technique uses advanced analytical platforms and techniques, such as gas-chromatography or high performance liquid chromatography coupled with mass spectrometry (GC-MS, HPLC-MS), Magnetic resonance (NMR), capillary electrophoresis (CE), which are improved continuously to identify constellations, patterns of metabolites related to specific pathways and pathologies [13].

Based on recent literature findings, this review focuses on the description of best methods and procedures to separate and identify hydrophobic molecules with molecular weights under 1000 Daltons, especially using liquid chromatography coupled with mass spectrometry. The changes of the turnover of these molecules in tissues or blood may reflect an accurate diagnosis of CRC. The main lipid classes involved in the progression of CRC are also presented, as well the alterations of lipid-related cellular pathways during tumorigenesis.

\section{LIPID CLASSES AS PUTATIVE CRC BIOMARKERS}

Lipids are a very complex category of metabolites, including molecules with multiple structures and roles in cellular signaling and energy fuels [14]. Lipids are considered potential biomarkers in malignancy, therefore lipidomic studies are strongly developed the last two decades, helping the understanding, diagnosis and treatment of cancer and meanwhile to be good prognostic or screening biomarkers for early diagnosis and targeted therapies [15].

Figure 1 represents the main classes of lipids which may be considered as diagnosis biomarkers of many pathologic disturbances.

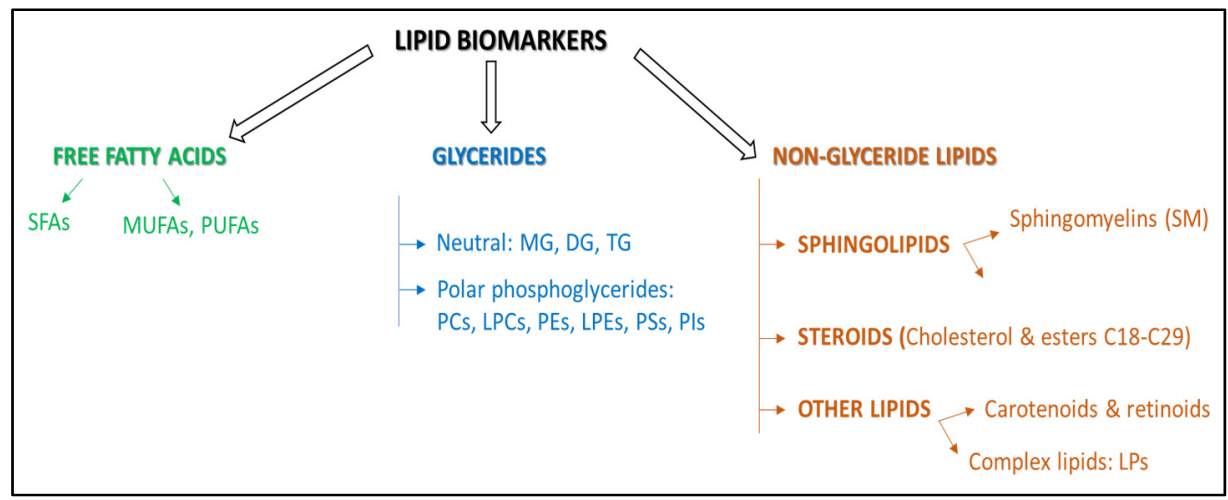

Figure 1. Main lipid classes, considered as potential biomarkers for many pathologies, including CRC. For details see abbreviations. 
The lipid alterations are associated with colorectal cancer (CRC), since cancer cells can generate energy in a nutrient-deficient environment, prefer glycolysis against oxidative phosphorylation. However, recently this paradigm shifted towards a "reversed Warburg effect", some cancer cells, including CRC cells may synthesize ATP by of mitochondrial phosphorylation $[16,17]$, opening new research directions for the identification of molecular therapeutic targets, such as fatty acids (FAs) synthesis and oxidation. The metabolization of exogenous glutamine represents another dependence of cancer cell, many oncogenic mutations affecting glutamine metabolism [18]. Meanwhile the alterations of lipid metabolism in CRC lead to structural changes in cell membranes, disruption of energy homeostasis, cell signaling, gene expression and protein distribution, affecting a number of cell functions, such as proliferation, differentiation, apoptosis, autophagy, necrosis, drug and chemotherapy resistance $[19,20]$. The role of lipids and their metabolism in cancer development and spread raises a growing interest of researchers, as shown in previous reviews $[1,21]$. The lipid metabolic pathways affected in CRC cells include FAs synthesis, desaturation, elongation and mitochondrial oxidation of the FAs. A summary of the main changes in the lipid composition of different biological samples from CRC patients, as shown in recent references, is presented in Table 1.

Table 1. Changes in lipid composition of different biological samples from CRC patients

\begin{tabular}{|l|l|l|l|}
\hline $\begin{array}{c}\text { Biological } \\
\text { sample }\end{array}$ & \multicolumn{1}{|c|}{ Lipid species/potential biomarkers } & \multicolumn{1}{|c|}{$\begin{array}{c}\text { Change } \\
\text { tendency }\end{array}$} & References \\
\hline \multirow{5}{*}{ Tissue } & PCs, especially PC(16:0/16:1) & Increase & {$[22]$} \\
\cline { 2 - 4 } & Total lipids, Cerotic acid & Increase & {$[23-25]$} \\
\cline { 2 - 4 } & TAGs & Decrease & {$[26,27]$} \\
\cline { 2 - 4 } & Lipoxygenase & Increase & {$[28]$} \\
\cline { 2 - 4 } & FFAs 22:4 & Increase & {$[21,29,30]$} \\
\cline { 2 - 4 } & Ceramides and endo cannabinoid & Decrease & {$[29]$} \\
\cline { 2 - 4 } & LPC 20:4,LPA & Increase & {$[31]$} \\
\cline { 2 - 4 } & LPC 22:6 & Decrease & {$[31]$} \\
\cline { 2 - 4 } & PA 34:0, 36:2 & Increase & {$[30,31]$} \\
\hline \multirow{5}{*}{ Plasma } & LPC & Increase & {$[25,31,32]$} \\
\cline { 2 - 4 } & Fatty acids synthesis & increase & {$[33-35]$} \\
\cline { 2 - 4 } & MUFAs/PUFAs & Increase & {$[30]$} \\
\cline { 2 - 4 } & Ethanolamine plasmalogens & {$[36,37]$} \\
\cline { 2 - 4 } & fatty acids and polar lipids & Increase & {$[38]$} \\
\cline { 2 - 4 } & Decanoic acid & Decrease & {$[39]$} \\
\cline { 2 - 4 } & LPC18:0 & Decrease & {$[23,40,41]$} \\
\hline \multirow{2}{*}{ Serum } & Total lipids C15:0, C18:0, C18:0,C18:2, C18:3 & & \\
\hline
\end{tabular}


LIPIDOMICS: ADVANCED ANALYTICAL TECHNOLOGY TO IDENTIFY BIOMARKERS OF COLORECTAL CANCER

\begin{tabular}{|l|l|l|l|}
\hline $\begin{array}{c}\text { Biological } \\
\text { sample }\end{array}$ & \multicolumn{1}{|c|}{ Lipid species/potential biomarkers } & \multicolumn{1}{c|}{$\begin{array}{c}\text { Change } \\
\text { tendency }\end{array}$} & References \\
\hline \multirow{5}{*}{$\begin{array}{l}\text { Total lipids C22:0, C24:0, C26:0, C26:0, C30:0, } \\
\text { C18:1 }\end{array}$} & Increase & {$[23,41]$} \\
\cline { 2 - 4 } & Ultralong chain fatty acids & Decrease & {$[42]$} \\
\cline { 2 - 4 } & Oxilipins, HODE, HETE & Decrease & {$[43]$} \\
\cline { 2 - 4 } & Endocannabinoids and ceramides & Increase & {$[44]$} \\
\cline { 2 - 4 } & Sphingomyelins, Ceramides, eicosanoids & Increase & {$[45-47]$} \\
\cline { 2 - 4 } & GTA-446 & Increase & {$[48]$} \\
\cline { 2 - 4 } & $\begin{array}{l}\text { Succinate, N2, N2-dimetihylguanosine, adenine, } \\
\text { citraconic acid,methylguanosine }\end{array}$ & Increase & {$[49]$} \\
\hline
\end{tabular}

The complex lipid metabolic changes may be explained by the high proliferation rate of the CRC cells, with high energetic needs, changes in the serum levels of phospholipid components derived from cell membrane degradation, accompanied by inflammation and changes in the arachidonic acid metabolites in serum or tissue.

A recent study focused on a single metabolite as a potential biomarker for early detection of CRC, namely long-chain (C28) hydroxylated polyunsaturated fatty acids or gastrointestinal tract acids (GTAs). Analyzing serum samples from 225 CRC patients and 916 healthy volunteers, it was concluded that GTA-446 does not reflect the presence of a tumor, but the susceptibility to CRC and helps to identify high-risk CRC patients with high sensitivity, encouraging the colonoscopy [46].

Another study identified decreases of LPCs, associated with body weight loss and activated inflammatory status in CRC patients [50]. Another 2019 published paper, based on the study of plasma form 268 CCR patients and 353 controls, finds all the LPC positively correlated as it follows: seven LPCs were detected at lower levels among colorectal cancer patients compared to controls, mainly LPC (16:0 and 18:0), in accordance with previous studies. This pattern might reflect an increased degradation rate of LPCs as a result of the accelerated cell proliferation (LPEs20:4 and 22:6) were increased in CRC patients compared to controls [51,52].

An important aspect of CRC tumorigenesis is related to the eicosanoids (arachidonic acid metabolites) and their oxidized forms via cyclooxygenase2 and 15- lipoxygenase-1 activities, these enzymes having opposite effects: the first one generates prostaglandin E2 which stimulates tumorigenesis while the second one inhibits interleukin-1 and tumor necrosis factor TNFa and suppresses colonic tumorigenesis [48]. Meanwhile 13-HODE levels were significantly decreased in cancer and colorectal polyp mucosa, as a specific alteration in the LOX product profile associated with human colorectal tumorigenesis. No significant differences between normal, polyp and cancer mucosa were noticed for 12-HETE, 15-HETE or leukotriene B4 levels [28]. 


\section{LIPID BIOMARKERS IN RELATION TO CRC DIAGNOSIS AND STAGE}

In 2012, in a study including 52 CRC patients and 52 healthy controls there were 15 metabolites identified categorized in four lipid classes. The authors found a lipid metabolic model containing palmitic amide, oleamide, octadecanoic, hexadecanedioic, myristic and eicosatrienoic acids, lysophosphatidylcholines such as LPC(16:0), LPC(18:2), LPC(20:4), LPC(22:6) with a good statistics as potential biomarkers (AUROC value of 0.991 ) to discriminate early stage patients from healthy controls, above the prediction made by carcinoembryonic antigen (CEA) [31].

The up-regulated and down-regulated metabolites through the various stages of CRC were identified in a study which included 56 CRC patients, 60 healthy controls and 59 patients with adenomas. Benzoic acid showed high AUROC value, octanoic and decanoic acids were up-regulated and proportional to CRC stage, but their diagnostic predictability was not high [47].

A study on plasma samples of $30 \mathrm{CRC}$ patients revealed changes of medium-chain fatty acids ( 6 up to $12 \mathrm{C}$ ) while a targeted metabolomic approach of further 117 patients revealed that decanoic acid (C10:0) had the best AUROC values in discriminating CRC patients from healthy individuals and may prove an excellent biomarker for screening [38].

A panel of 13 metabolites for the discrimination between CRC patients and healthy controls as well as 14 biomarkers for the discrimination between $\mathrm{CRC}$ and benign polyp patients, many of them derived from lipid metabolism: glyceraldehyde, hippuric acid, linolenic acid, glycochenodeoxycholate, glycocholate were recently reported [53]. Adding to the metabolic model four clinical factors (age, gender, smoking and alcohol consumption), the AUROC scores reached values of $0.93-0.95$, in differentiating CRC patients from healthy controls or polyp patients respectively. Another study showed that $\beta$-hydroxybutyrate increased continuously from stage I to stage IV CRC while tryptophan and indole acrylic acid were decreasing continuously [54-56].

\section{PREDICTIVE VALUE OF LIPID METABOLITES}

The main predictive factor for $\mathrm{CRC}$ is considered to be the stage of diagnosis [57] underlying the importance of CRC screening and early diagnosis. A Japanese study reported a prediction model for $60 \mathrm{CRC}$ patients comparative to 60 controls which included 4 metabolites (2-hydroxybutyrate, aspartic acid, kynurenine, and cysteamine) that showed better statistical power (AUROC, sensitivity, specificity and accuracy above 0.9 and $85 \%$ respectively) higher than CEA or CA 19.9 [58]. 
Using an MRI technique another model of 8 lipid and non-lipid metabolites (pyruvic, fumaric and glycolic acid, palmitoleic acid, ornithine, lysine tryptophan and 3-hydroxyisovaleric acid showed significant results [59]. Yang et al. [60] reported a 9 metabolites model that can predict the response to neoadjuvant chemotherapy for CRC. Metabolic profiles of 30 non-response patients were compared to those of 27 response cases and 6 metabolites related to lipid metabolism were found: Dioleoyl lecithin, 11-Keto-beta-boswellic acid, CE(18:2), SM(d16:1/18:1) and PCs 18:1/2:0 and16:1/22:6). All these lipids were upregulated in the response group compared with non-response group.

Another study [44] analyzed serum fingerprints from 20 CRC patients prior to surgery and one month after the radical intervention, compared to 20 healthy volunteers. The potential biomarkers were lipid classes PCs, LPCs and DGs, with low statistical difference between the pre-operative and postoperative status. One study developed in China and the United States found a common 15 metabolite pattern that could allow assessment of the CRC recurrence rate and survival of the patients after surgical intervention or chemotherapy. Using GC-MS of 376 surgical tissue specimens from four centers a panel of 15 metabolites was selected, able to distinguish between CRC patients having better prognostic outcomes, i.e., longer time-to-recurrence (52.9 vs 25.9 months), and better 5 -year survival rate (67.0 vs 44.7 months), earlier recurrence and lower survival rates. Out of these 15 metabolites, 4 were lipid molecules: glycerol, myristate, palmitoleate, 2-aminobutyrate [24].

\section{PROSPECTIVE STUDIES}

Recently, a relevant serum MS study for lipophilic metabolites made on 66 patient-control pairs, under the European Prospective Investigation into Cancer and Nutrition (EPIC), reported 9 metabolites to be related to CRC etiology and were recommended for further CRC prospective studies. Four other features seem to be involved in disease progression (reverse causality), as a potential, valuable biomarkers for early CRC detection, e.g. ultralong chain fatty acids which are decreased in the serum sample of CRC patients [42].

In another prospective study published in 2018 that included 250 incident cases with CRC 35 metabolites were found associated with CRC risk, including 12 glycerophospholipids, 9 decreasing, 3 increasing the CRC risk. The dysregulation of glycerophospholipids might have an important role in the risk of developing colorectal cancer [51].

A German prospective study, with average follow-up of 8.3 years, investigated the association between the pre-diagnosis levels of 120 metabolites and the risk of breast, prostate and colorectal cancer. The study included a 
subset of 163 CRC patients from over 25000 population of EPIC-Heidelberg study. It was concluded that changes in plasma lipid composition precede the appearance of neoplasia for several years and that tumor changes can cause a global change in LPC metabolism. It seems that the high level of LPC 18:0 (unsaturated fatty acids) would be a protective factor [39].

\section{LIPID BIOMARKERS TO DISTINGUISHING BETWEEN ADENOMAS AND MALIGNANT TUMORS}

Using ${ }^{1} \mathrm{H}-\mathrm{NMR}$ spectrometry, the blood serum of $40 \mathrm{CRC}$ patients, 32 polyp patients and 38 healthy controls was analyzed. Glutamine, succinate, glycerol, aspartate, and lactate were the potential non-lipid biomarkers in the colorectal polyp group data, while in the CRC patients' group, lactate, glycine, glutamate, glutamine, and aspartate were found as potential biomarkers. The authors observed similarities and differences between the metabolism of colorectal polyps and CRC patients, but the level of choline was significantly elevated in both CRC and colorectal polyp groups. On the other hand, 3-hydroxybutyrate, PUFAs and glycerol (from the glycerolipid metabolism), were abnormal in the colorectal polyp [45]. Regarding the specificity of lipid metabolites there are some similarities and differences between colorectal polyps and CRC, compared with healthy volunteers. Another group found in serum samples that glycerolipid metabolism was modified in the colorectal polyp (higher levels of lipids and PUFAs and lower level of glycerol) [61]. The authors draw the possible conclusion that glycerolipid metabolism is involved in the ATP generation. The explanation for increased levels of choline-related metabolites in tumors is probably the result of accelerated lipid membrane metabolism involved in the ATP generation, due to rapid cell proliferation. Glucose changes were consecutive to glycolysis, upregulated in CRC while increases of 3-hydroxybutyrate, an end metabolite of fatty acids suggests the upregulation of fatty acid $\beta$-oxidation needed as energy support cancer cell proliferation [45]. Increased oxidative stress is usually associated with increased oxidation of fatty acids, which may result in an accumulation of 3-hydroxybutyrate [24].

\section{RELATIONSHIPS BETWEEN CRC AND OTHER METABOLIC DISEASES}

There are some question marks about the specificity of above-mentioned biomarkers related specifically to $\mathrm{CRC}$, or if these lipid metabolites are generally characteristic to tumoral processes or are influenced by other dysmetabolic 
diseases (e.g. metabolic syndrome - obesity or diabetes). When the metabolic pathway differences between visceral and subcutaneous adipose tissue were studied in CRC patients by metabolomic and transcriptomic methods, increases of proinflammatory lipid metabolism was observed, increase of free arachidonic acid, activation of phospholipases and prostaglandin synthesisrelated enzymes [62].

Many articles demonstrated positive relationships between obesity, diabetes, dyslipidemia and CRC. In the etiopathogenesis of CRC in obese/ over-weight patients, fat tissue is very important in tumorigenesis, since it acts like glandular tissue, develop endocrine, paracrine and autocrine functions, controlling triglyceride metabolism, affecting the coagulation system and suppressing the anti-lipolytic action of insulin [63]. The literature data regarding the correlation between serum cholesterol, the triglycerides levels and the risk of CRC are not homogenous. In a large-scale cohort study, increased serum TGs levels were associated with higher risk of rectal cancer, as well as in lung, gynecological cancers and thyroid cancer, but not in colon cancer [64]. Another study showed good positive correlation between TG level and CRC, modest positive correlation between total cholesterol and CRC in male patients but not in women [65].

Meanwhile the levels of polar metabolites like nucleotides, nucleosides, and their metabolites, branched chain and aromatic amino acids have been correlated positively or negatively with obesity and diabetes, whereas glutamine and glycine levels are decreased, depending on the degree of insulin resistance in obese subjects. Serum concentrations of LPCs had inverse correlation with the body mass index, body weight and waist and hip circumference and treatment with metformin decreases the CRC risk in diabetic patients [66].

Men with type 2 diabetes mellitus have important risk in developing proximal CRC while in women no significant correlation was found $[60,67,68]$. Also, the particular association of fatty acids, high levels of linoleic acid, oddchain and very long-chain fatty acids was correlated with a decreased incidence of type 2 diabetes and influenced by metabolic, genetic and nutritional factors $[69,70]$.

\section{CONCLUSION}

The recent evolution of metabolomics as a technology to investigate beyond fingerprints and biomarkers, the mechanisms of CRC evolution [71, 72] was diversified towards lipidomic profile, which already proved a great potential as a high value for a proper metabolic signature of tissues and biofluids from CRC patients and to discriminate significantly between healthy 
controls, benign polyps versus malignant tumors [73]. Specific classes of lipids involved in cellular signaling and energy provision proved to be good biomarkers for CRC in different stages, during treatment monitoring or as prognosis factors.

Integrated approaches using advanced ultrahigh performance liquid chromatography-high resolution mass spectrometry revealed the deep involvement of lipid molecules in CRC pathology [74, 75]. Lipid profile alterations in particular, e.g. presence of cerotic acid or a decrease in hydroxylated, polyunsaturated long-chain fatty acids, an increase short free fatty acids, oxylipins, glycerophospholipids, di- and triglycerides, sphingomyelins and ceramides showed statistically significant differences and can be considered reliable biomarkers, differentiating between early and advanced stages of this malignancy or serving as survival predictors. The complex data base Lipid offers a great help for the identification of lipid biomarkers in CRC diagnosis and monitoring. Advanced methodology and studies on larger cohorts of patients are still needed for the development of clinically useful lipid biomarkers.

\section{REFERENCES}

1. R.L. Siegel; K.D. Miller; S.A. Fedewa; D.J. Ahnen; R.G.S. Meester; A Barzi; Cancer J. Clin., 2017, 67, 177-179.

2. M.S. Cappell. Gastroenterol Clin N Am., 2008, 37, 1-12.

3. D.W. Sheneman; J.L. Finch; W.A. Messersmith; S. Leong; K.A. Goodman; S.L. Davis; Colon Cancer., 2017, 6, 49-56.

4. B.K. Edwards; E. Ward; B.A. Kohler; C. Eheman; A. G. Zauber; R. N.Anderson; L. A. G. Ries; Cancer, 2010, 116, 544-557.

5. Pakiet; J. Kobiela; P. Stepnowski;T.Sledzinski; A.Mika; Lipids Health Dis., 2019, 18, 29-38.

6. A.E. Obaro; D.N. Burling; A.A. Plumb; Br. J. Radiol., 2018, 91, art. 20180307

7. Y. Nasseri; S.J. Langenfeld;.Surg. Clin. North Am., 2017, 97, 503-513.

8. J. Zhang; Z. Cheng; Y Ma; C. He; Y. Lu; Y. Zhao; X. Chang; Y. Zhang; Y. Bai; N. Cheng; Clin Colorectal Cancer, 2017, 16, 252-263.

9. U. Ladabaum; J.A. Dominitz; C. Kahi; R.E. Schoen; Gastroenterology, 2020, 158, 418-432.

10. P.J. Pickhardt; Radiol. Clin. North Am., 2017, 55, 1183-1196.

11. D.S. Wishart; R. Mandal; A. Stanislaus; M. Ramirez $\urcorner$ Gaona; Metabolites, 2016, 6, 10-21.

12. L. D. Roberts; A. L. Souza; R. E. Gerszten; C. B. Clish; Targeted metabolomics. Curr. Protocols in Mol. Biol., 2012, 1(SUPPL.98), Chapter 30, Unit 30-30.2.24

13. A.D. Beggs; M. P. Dilworth; Br. J. Surgery, 2015, 102, e29-40. 
14. E. Fahy; D. Cotter; M. Sud; S. Subramaniam; Biochim. Biophys. Acta-Mol. Cell Biol. Lipids, 2011, 1811, 637-647.

15. D.J. Stephenson; L.A. Hoeferlin; C.E. Chalfant; Transl. Res.,2017,189,13-29

16. X.D. Xu; S.X. Shao; H.P. Jiang; Y.W. Cao; Y.H. Wang; X.C. Yang; Oncol. Res. Treat., 2015, 38,117-122.

17. V. Chekulayev; K. Mado; I. Shevchuk; A. Koit; A. Kaldma; A. Klepinin, Biochem. Biophys. Reports, 2015, 4, 11-25.

18. Y. Hao; Y. Samuels; Q. Li; D. Krokowski; B-J. Guan; C. Wang;Nat.Commun. 2016, 7, art.11971

19. C. Huang; C. Freter; Int. J. Mol. Sci., 2015, 16, 924-949.

20. Y.Y.Zaytseva; J.W. Harris; M.I. Mitov; J.T. Kim; D.A. Butterfield; E.Y. Lee; Oncotarget, 2015, 6, 18891-904.

21. Currie E, Schulze A, Zechner R, Walther TC, Farese RV Jr.; Cell Metab., 2013,18, 15

22. N. Kurabe; T. Hayasaka; M. Ogawa; N. Masaki; Y. Ide; M. Waki;. Cancer Sci., 2013, 104, 1295-1302

23. Mika; J. Kobiela; A. Czumaj; M. Chmielewski; P. Stepnowski; T. Sledzinski; Cell Physiol. Biochem., 2017, 41, 722-730.

24. Y. Qiu; G. Cai; B. Zhou; D. Li; A. Zhao; G. Xie; H. Li; Clin. Cancer Res., 2014, 20, 2136-2147.

25. Q. Zhang; H. Xu; R. Liu; R. Gao; X. Yang; W. Jin; Y. Zhang; K. Bi; Q. Li; Anal. Chem., 2019, 91, 3389-3396.

26. Mika; A. Pakiet; A. Czumaj; Z. Kaczynski; I. Liakh; J. Kobiela; A. Perdyan; K. Adrych; W. Makarewicz; T. Sledzinski; J. Clin. Med., 2020, 9, 1095-1105.

27. T. Liu; F. Peng; J. Yu; Anal. Bioanal. Chem., 2019, 411, 5079-5088.

28. Shureiqi; D. Chen; R. S. Day; X. Zuo; F. L. Hochman; W. A. Ross; S. M. Lippman; Cancer Prevention Res., 2010, 3, 829-838.

29. L. Chen; H. Chen; Y. Li; L. Li; Y. Qiu; J. Ren; Oncol Rep., 2015b, 34, 447- 454.

30. A. S. Guo; Y. Wang; D. Zhou; Z. Li; Sci Rep., 2014, 4, 5959.

31. S. Li; B. Guo; J.W. Song; X.L. Deng; Y.S. Cong; P.F. Li; Metabolomics, 2013, 9 , 202-212.

32. Z. Zhao; Y. Xiao; P. Elson; H. Tan; S.J. Plummer; M. Berk; J Clin Oncol. 2007, $25,2696-2701$.

33. M. Okuno; K. Hamazaki; T. Ogura; H. Kitade; T. Matsuura; R. Yoshida; In Vivo, 2013, 27, 203-210.

34. L.M. Butler; J-M. Yuan; J.Y. Huang; J.Su; R. Wang; W-P. Koh; Precis Oncol., 2017, 1, 38.

35. J. Zhang; L. Zhang; X. Ye; L. Chen; L. Zhang; Y. Gao; Prostaglandins Leukot Essent Fat Acids., 2013, 88, 355-360.

36. S. Shen; L. Yang; L. Li; Y. Bai; C. Cai, H. Liu; J. Chromatography B, 2017, 10681069, 41-48.

37. T. Liu; Z. Tan; J. Yu; F. Peng; J. Guo; W. Meng; Y. Chen; T. Rao; Z. Liu; J. Peng; Expert rev. Proteomics, 2020, 17, 233-242.

38. S. Crotti; E. Agnoletto; G. Cancemi; V. Di Marco; P. Traldi; S. Pucciarelli; M. Agostini; Anal. Bioanal.Chem., 2016, 408, 6321-6328. 
CLAUDIU RĂCHIERIU, DAN T. ENIU, EMIL MOIŞ, FLORIN GRAUR,

CARMEN SOCACIU, MIHAI ADRIAN SOCACIU, NADIM ALHAJJAR

39. T. Kühn; A. Floegel; D. Sookthai; T. Johnson; U. Rolle-Kampczyk; W. Otto; R. Kaaks; BMC Medicine, 2016, 14, 13-26.

40. J. Zhu; D. Djukovic; L. Deng; H. Gu; F. Himmati; E.G. Chiorean; J. Proteome Res., 2014, 13, 4120-4130.

41. Y. Kondo; S. Nishiumi; M. Shinohara; N. Hatano; A. Ikeda; T. Yoshie; Biomark Med., 2011, 5, 451-460.

42. K. Perttula; C. Schiffman; W. M. B. Edmands; L. Petrick; H. Grigoryan; X. Cai; S. M. Rappaport; BMC Cancer, 2018, 18, 996-1010.

43. L. Zhang; B. Chen; J. Zhang; J. Li; Q. Yang; Q. Zhong; Prostaglandins Leukot Essent Fat Acids, 2017, 120, 25-31.

44. L. Chen; C. Zhang, Q. Gui; Y. Chen; Y. Yang; Molecular Medicine Reports, 2015, $12,6879-6886$.

45. J.H. Jung; K. Taniguchi; H.M. Lee; R. Bandu; K. Komura; Sci. Rep., 2020, 10, art nr.6124.

46. X. Zuo; I. Shureiqi; Prostaglandins and Other Lipid Mediators, 2013, 104-105; 139-143K. Uchiyama; N. Yagi; K. Mizushima; Y. Higashimura; Y. Hirai; T. Okayama; Y. Naito; J. Gastroenterol., 2017, 52, 677-694.

47. J. Zhu; D. Djukovic; L. Deng; H. Gu; F. Himmati; M. Abu Zaid; D. Raftery; Anal. Bioanal. Chem., 2015, 407, 7857-7863.

48. T. Hata; I. Takemasa; H. Takahashi;N.Haraguchi; J. Nishimura; Br. J. Cancer, 2017, 117, 227-232.

49. I. Tan; Y. Qiu; X. Zou; T. Chen; G. Xie; Y. Cheng; W. Jia; J. Proteome Res., 2013, 12, 3000-3009.

50. X. Shu; Y. B. Xiang; N. Rothman; D. Yu; H. L. Li; G. Yang; W. Zheng; Int. J. Cancer, 2018, 143, 527-534.

51. J. M. R. Geijsen; S. Brezina; P. Keski-Rahkonen; A. Baierl; T. Bachleitner-Hofmann; M. M. Bergmann; C. M. Ulrich; Int. J. Cancer, 2019, 145, 1221-1231.

52. D. Djukovic; J. Zhang; D. Raftery; Colorectal cancer detection using targeted LC-MS metabolic profiling. In: Methods in Molecular Biology, 2018, 1765, 229240 Humana Press Inc.

53. F. Farshidfar; A. M. Weljie; K. Kopciuk; W. D. Buie; A. Maclean; E. Dixon; O.F. Bathe; Genome Med., 2012, 4, article nr. 42

54. F. Farshidfar; A. M. Weljie; K. Kopciuk; S. E. Hilsden; F. Mcgregor; W. D. Buie; O. F. Bathe; Br. J. Cancer,m2016, 115, 848-857.

55. F. Farshidfar; K.A. Kopciuk; R. Hilsden; S.E. Mcgregor; V.C. Mazurak; W.D. Buie; BMC Cancer, 2018, 18, 26-36.

56. American Cancer Society. Colorectal Cancer Facts \& Figures 2020-2022 Atlanta: American Cancer Society Inc., 2020, 32 pg.

57. S. Nishiumi; T. Kobayashi; S. Kawana; Y. Unno; T. Sakai; K. Okamoto; M. Yoshida; Oncotarget, 2017, 8, 17115-17126.

58. S. Nishiumi; T. Kobayashi; A. Ikeda; T. Yoshie; M. Kibi; Y. Izumi; M. Yoshida; PLOS ONE; 2012, 7, e40459.

59. K. Yang; F. Zhang; P. Han; Z. Wang; K. Deng; Y. Zhang; Z. J. Zhu; Metabolomics, 2018, 14, article nr. 110. 
60. J. Gu; Y. Xiao; D. Shu; X. Liang; X. Hu; Y. Xie; D.Lin; H. Li; Dis. Markers, 2019, ID 3491852.

61. D. B. Liesenfeld; D. Grapov; J. F Fahrmann; M. Salou; D. Scherer; R.Toth; N. Habermann; J. Böhm; P. Schrotz-King; B. Gigic; M. Schneider; A. Ulrich; E. Herpel; P. Schirmacher; O. Fiehn; J.W. Lampe; C.M. Ulrich; Amer. J. Clin. Nutr., 2015, 102, 433-443.

62. M. Muc-Wierzgoń; E. Nowakowska-Zajdel; S. Dzięgielewska-Gęsiak; T. Kokot; World J. Gastroenterol.,2014, 20, 9759-9774

63. H. Ulmer; W. Borena; K. Rapp; J. Klenk; A. Strasak; G. Diem; G. Nagel; Br. J. Cancer, 2009, 101, 1202-1206.

64. S. Strohmaier; M. Edlinger; J. Manjer; T. Stocks; T. Bjørge; W. Borena; H. Ulmer; PloS One, 2013, 8, e54242.

65. O. D. Rangel-Huerta; B. Pastor-Villaescusa; A.Gil; Metabolomics, 2019, 15, art nr. 93.

66. P. J. Limburg; R. A. Vierkant; Z. S. Fredericksen; C. L. Leibson; R. A. Rizza; A. K. Gupta; J. R. Cerhan; Am. J. Gastroenterol., 2006, 101, 1872-1879.

67. S-W. Oh; Y.-H. Kim; Y. S. Choi; D. K. Chang; H. J. Son; P-L. Rhee; S. G. Shim; Diseases of the Colon and Rectum, 2008, 51, 56-61.

68. F. Imamura; S. J. Sharp; A. Koulman; M. B. Schulze; J. Kröger; J. L. Griffin; N. J. Wareham; PLoS Medicine, 2017, article nr. 1410.

69. Y. X. Yang; S. Hennessy; J. D. Lewis; Clin. Gastroenterol. Hepatol., 2005, 3, 587-594.

70. C. Johnson; J. Ivanisevic; G. Siuzdak; Nat. Rev Mol. Cell Biol., 2016, 17, $451-$ 459.

71. F. Zhang; Y. Zhang; W. Zhao; K. Deng; Z. Wang, Oncotarget, 2017, 8, 35460-35472.

72. F. Yan; H. Zhao; Y. Zeng; Clin. Trans. Med., 2018, 7, 21-29.

73. G. Charkoftaki; D.C. Thompson; J.P. Golla; R. Garcia-Milian; T.K.T. Lam; J. Engel; Chem. Biol. Interact., 2019, 304, 88-96.

74. Triebl; M. Trötzmüller; J. Hartler; T. Stojakovic; H.C. Köfeler; J. Chromatogr. B., 2017, 1053, 72-80.

75. Y. Wang; S. Hinz; O. Uckermann; P. Hönscheid; W. von Schönfels; G. Burmeister; A. Hendricks; Biochim. Biophys. Acta, Mol. Cell Biol. Lipid, 2020, 1865, 158579. 